\title{
Influence of photomodulation of the structural and energetically state of water and its biological activity
}

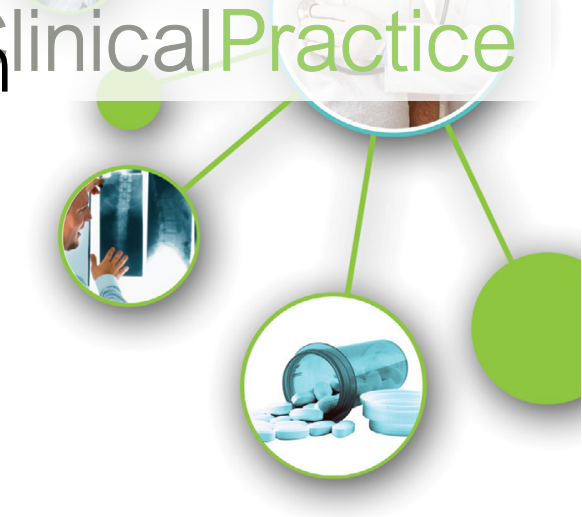

\begin{abstract}
The paper provides an assessment of changes in the structural and energetic state of the associated water phase under the light radiation influence on the direction of its regulatory influence on the various human body systems. Based on the evaluation of the regulatory response of these body systems and the behavior of the biotest object, Escherichia coli K12 TG1, the associated water phase resonant states, which have a targeting effect on these human body systems and the control test object, will be established. The shifts of resonant (tonic and sedative) phase states under the influence of water photomodulation are estimated. The photomodulation influence establishes the long-term dynamics of changes in the structural and energetic state of the associated water phase, including the advanced current time, correlating with the reactions of the human body systems.
\end{abstract}

Keywords: The associated water phase, photobiomodulation, non-local dynamics of the electronic state, test system of $E$. Coli microorganism, human body systems

Abbreviations: ER: Electromagnetic Radiation; UHF: Ultra-High Frequency; HF: High Frequency; LFE: LowFrequency Emission; NIR: Non-lonizing Radiation; CNS: Central Nervous System; CVS: Cardiovascular System; GIT: Gastrointestinal Tract

\section{Introduction}

According to current perspectives, the mechanism of radio frequencies and red range radiations action, including the infrared and UHF ranges, is associated with their interaction with proteins and nucleic acids charges, leading mainly to hydrogen bonds destabilization in them and conformation changes [1], accompanied by the genes activation [2]. The above effects of the radiation biological action do not contradict the concept of the primary cellular regulation linkin terms of the associated water phase $[3,4]$ but are its natural consequence.

With this natural consequence, the transformation of the associated water phase under photomodulation is macroscopically nonlocal [3,5], which, in turn, is manifested in a long after effect of water recorded by the body systems reaction and in the effects of strengthening the body systems response to the remote effect associated with the interference of de Broglie partial components. When establishing the mechanism of the electromagnetic radiation effect on the body, it is necessary to consider the high sensitivity of the associated water phase the to radiation in a wide range of wavelengths from ultra-low (less than $1 \mathrm{~Hz}$ ) to $\mathrm{HF}, \mathrm{UHF}$ and visible frequency bands, which are all related to the phase superconducting state $[3,6]$. In this regard, many of the body's responses, regardless of the wavelength range, have a similarity that depends more on the intensity of the radiation rather than on their frequency. Moreover, the regulatory effect of Electromagnetic Waves (EMW) is informational in nature, it is not the intensity that is important, but rather the effect of radiation on the water that matters [7].

In addition to the ultrahigh sensitivity of the associated water phase to radiation over a wide range of wavelengths that are not associated with their absorption in all ranges from ultralow frequencies, HF, UHF, and to the visible range of radiation, water exhibits a selective effect on living organisms. It is obvious that this water ability is associated with structural changes in the phase that occur under the radiation influence without introducing
Konstantin Pronko ${ }^{1}$, Vladimir Zemskov ${ }^{1 *}$, Anatoly Stekhin ${ }^{2}$, Galina Yakovleva ${ }^{2}$, Aleksandr Marasanov $^{2}$ and Aleksandr Karasev ${ }^{2 *}$

${ }^{1}$ Department of Research and Development at Facecontrol, Inc., Florida, Miami, USA

${ }^{2}$ Center for Strategic Planning and Control of Medical and Biological Risks to Health, Moscow, Russia

*Author for correspondence:

vzemskov@facecontrol.biz 
additional chemical compounds into the water, as it happens in homeopathy. As a result of the phase transformations, not only the frequencies of water generation activity in the LF, UHF and HF ranges vary, but the action directivity of the water associates on the body cellular structures varies [3].

In this connection, it becomes possible not only to determine the targeting effect of the associated water phases with biological structures but also to assess their contribution to the overall adaptation syndrome (specific adaptation) at its primary regulatory stage $[8,9]$.

The existing functional systems of the body are differentiated into groups on the basis of neurohumoral regulation [10]. However, the relationship between the primary body systems response to stress (antistress or eustress) and its inter-system redistribution followed by a generalized nonspecific adaptation reaction, detailed in the works of $\mathrm{H}$. Selye and other authors [8], does not consider the contribution and interrelation of external and internal adaptation factors. This is especially important for understanding the reasons for the imbalance of the body systems, especially systems with a low response rate, for which the probability of oppression and the functioning level by the dominant body system is higher [9]. This phenomenon was previously reflected as a consequence of Le Chatelier-Braun principle, namely, the principle of development inhibition: maximum inhibitory effects occur in the period of the greatest potential development rates of the system. The identification of the root causes the inhibition and human body systems toning and the establishment of their connection with the electronic associated water phase activity, which is the only external source of negative entropy for the cell, makes it possible to create the basis for implementing the programs for non-drug correction of the human body systems functional state.

\section{Research instruments and methods}

To reveal the connection between the regulatory function of the associated water phase in the cellular metabolism, we used a programmethodical complex. This included generator equipment based on photomodulation (Lenovo), a measuring complex for diagnosing the person functional state and their body systems activity
"Rhythm Express" [11] and equipment for measuring the activity of microorganisms "Ecolum" and for the parametrization of water structural and energetic state [12].

The photodynamic correction of human body systems is based on the well-known laws of biological activation in the long-wave part of the visible light (red, orange, yellow), which have a sympathetic-tonic effect. In the short-wave part of the spectrum (light blue, blue, violet) appears parasympathetic action. Hence, the green part of the spectrum matches both of these effects on the body [13].

The photomodulation program is a specially developed program designed to influence the water through the light radiation from a Lenovo mobile computer screen (Ideapad MIX 31010ICR) and acoustic radiation from the builtin and/or remote (headphones) of the speaker system (IMAGES 1 and 2). The use of a

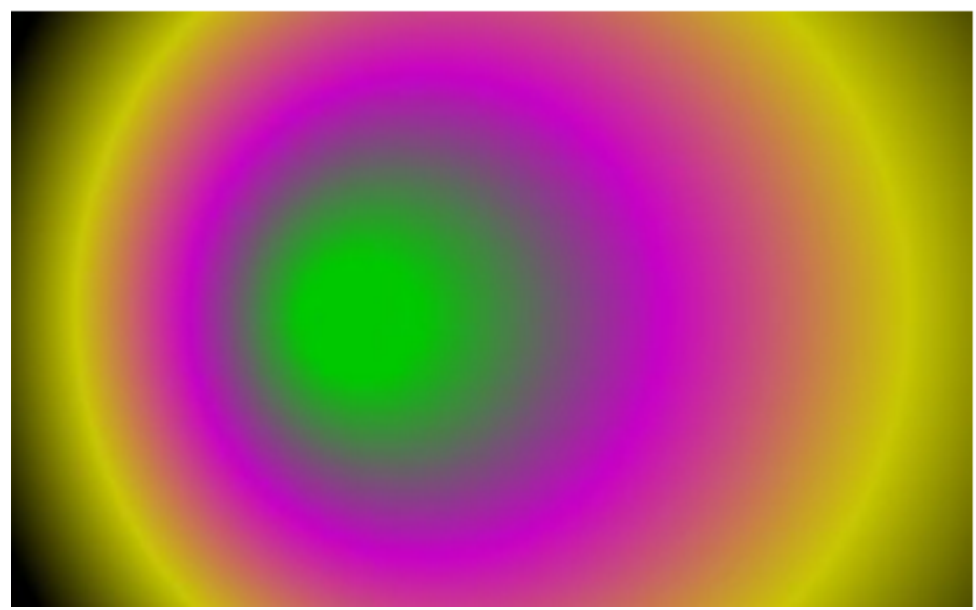

IMAGE 1. "Relax mode" program implements the color gamut of RGB coding: R0 -G255-B0, R255-G0-B255, R 255-G255-B0.

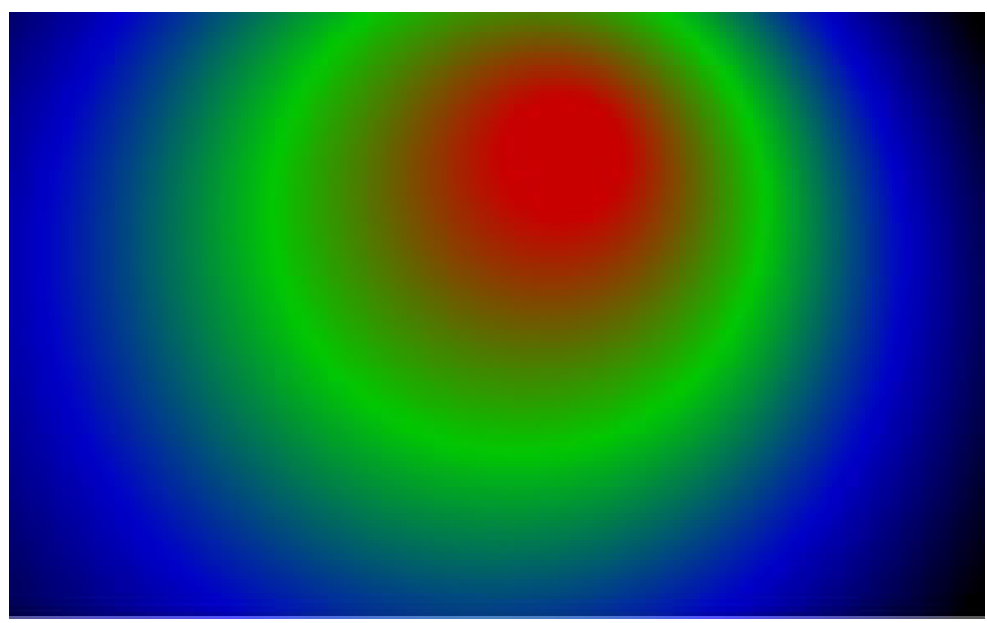

IMAGE 2. "Energe" program uses $671 \mathrm{~Hz}$ acoustic modulation along with the color gamut of RGB coding (R255-G0-B0, R0-G255-B0, R0-G0-B255). 
short-term (single) exposure of the body photo correction with blue light with a maximum activity at the wavelength of $-470 \mathrm{~nm}$ in the color range of the "Energy mode" program is aimed at improving a person's cognitive functions (mental activity, mood, attention) and maintaining his/her vigor with the simultaneous effect of the drowsiness blocking [14]. The physiological effect of a short-term exposure to blue light is associated with the passivation of certain brain areas that are responsible for the melatonin synthesis. This is illustrated by the delta-theta rhythms activity decrease (1-7 $\mathrm{Hz}$ ). However, there are restrictions on the body photostimulation with blue light. This is especially the case in the evening and at night, when the misalignment risks of circadian rhythms increase, which in turn can lead to sleep problems [15].

Supplementing blue light radiation with radiation in red and green wavelength ranges additionally ensures the biochemical processes (green) activation and microcirculation stimulation (blue-blue-green). Moreover, the blue and red light radiation synergistic effect reveal itself in the increasing the body resistance to pathogenic microorganisms, a vasodilating effect and the acceleration of recovery processes. Additionally, the red light radiation combination with shades of yellow exerts a tonic effect on the exposed human body skin [13].

Investigations of the water reaction to irradiation with electromagnetic radiation of the visible wavelength range under the "Relax mode" program were carried out using the instrumentmethodological complex for evaluating the structural and energetic water state. These investigations were based on the structural and energetic state parametrization methods of its associated phase and electrochemical indices [5].

Irradiated water, from 1 to 4 factory polymeric capacities of 0.5 liters each, was installed vertically on the monitor of the computer device and irradiated for 6 minutes. One week before the experiments, the used Nestle water was opened to establish oxygen equilibrium with the ambient air. This also allowed for the relaxation of the associated water phase excited states, which had been caused by changes in the electrochemical equilibrium resulting from the release of excess oxygen from the water.
Preliminary experiments showed that changes in the associated water phase state during its irradiation under the "Relax mode" program are not limited to short-period reactions, but also cover long for several days intervals. In this regard, to identify the changes emerging in the phase state, two weekly series of experiments were performed, and the results of these confirmed the above assumption. During the research, measurements of the water structural and physical state were carried out a day before the irradiation and after the water treatment (every hour under "Ecolum" test). The dynamics in "Nestle" water changes after its treatment was evaluated for the next several days. The structural and physical changes in irradiated water samples, a microcapillary cryophysical technique was used [12,16-18]. The water biotesting was used under the "Ecolum" method (luminescent microorganism E. Coli) for the identification of the relationship between changes in water and its biological activity. The test microorganisms reaction was measured daily every 0.5 hours between 09:00 to 13:00 hrs. The effect of photoactivated water on a human body, an analog of the testing method, discovered by R. Voll and Glaser-Turk, was used. This method of system activity testing is based on the fact that a human body can remotely (through de Broglie waves) perceive signals of very low frequency and intensity generated by a water and in turn, the body can change the functional state of its systems that are being influenced.

With technical specifications, such an assessment was carried out by using the "HealthExpress" functional diagnostics complex. The procedure for testing the state of human body systems consisted of a 2-minute ECG signal recording (with subsequent analysis of the heart rate variation) upon the patient's hand touching the reservoir with water. The study was conducted on a group of volunteers, consisting of 25 people of both sexes between the ages of 24 to 75 years.

\section{The study of changes in the water structural and energetic state upon its being photomodulated with radiation from a portable device "Lenovo" under "Relax mode"}

The developed program is designed for physiological relaxation of a human body. Relaxation (from Latin relaxation-relief, 
relaxation) is the state of rest associated with complete or partial muscle relaxation, accompanied by mental stress removal.

The objectives of the study:

1. To study the structural and physical, and energetic state of "Nestle" water after its photomodulation under the "Relax mode" program

2. To assess the structural and energetic changes influence in the state of the associated water phase on biological activity

3. To test the regulatory photomodulated water effect on human body systems

\section{Results}

Water structural and physical state after its treatment by radiation under the Relax mode program is characterized by a change in electrochemical parameters compared to untreated water (TABLE 1). The direction of their change is determined by the appearance low concentrations of peroxide associates in the system, which reflects the processes of the associate's structural organization transformation. Additionally, the water oxidation-reduction potential values increase by several tens of millivolts in comparison with the initial values ( 1 day before treatment). In timeseparated experiments (1 week), there are similar changes in the oxidation-reduction potential values. Thus, in 1 day after irradiation, Eh of water reaches its minimum values $\mathrm{Eh}=157.5 \mathrm{mV}$ - in the $1^{\text {st }}$ experiment, $E h=37.5 \mathrm{mV}$ - in the $2^{\text {nd }}$ and $\mathrm{Eh}=184.4 \mathrm{mV}$ - in the $3^{\text {rd }}$ experiment). Two hours before the appearance of Eh maximum values, the oxidation-reduction potential is also minimized in comparison with the values measured the day before the water irradiation (in the $1^{\text {st }}$ series as of $20.12 \mathrm{Eh}=133.7 \mathrm{mV}$, in the $2^{\text {nd }}$ series as of $26.12 \mathrm{Eh}=183.6 \mathrm{mV}$ against the values measured the day before or even 2 days before the water irradiation $-\mathrm{Eh}=182.3 \mathrm{mV}$ (as of 18.12) and $\mathrm{Eh}=217.7 \mathrm{mV}$ (as of 25.12), respectively). This confirms the reproducibility of the ongoing processes in the series when treating water with optical radiation.

Concurrently, it was observed that during a monthly exposure, there is a tendency towards the maximum of the chemiluminescence decrease compared with its initial state for 1-2 days before treatment (TABLE 1) and its gradual acidification. This process reflects the long-term tendency of the associated water phase transformation.

The changes in the electrochemical indices

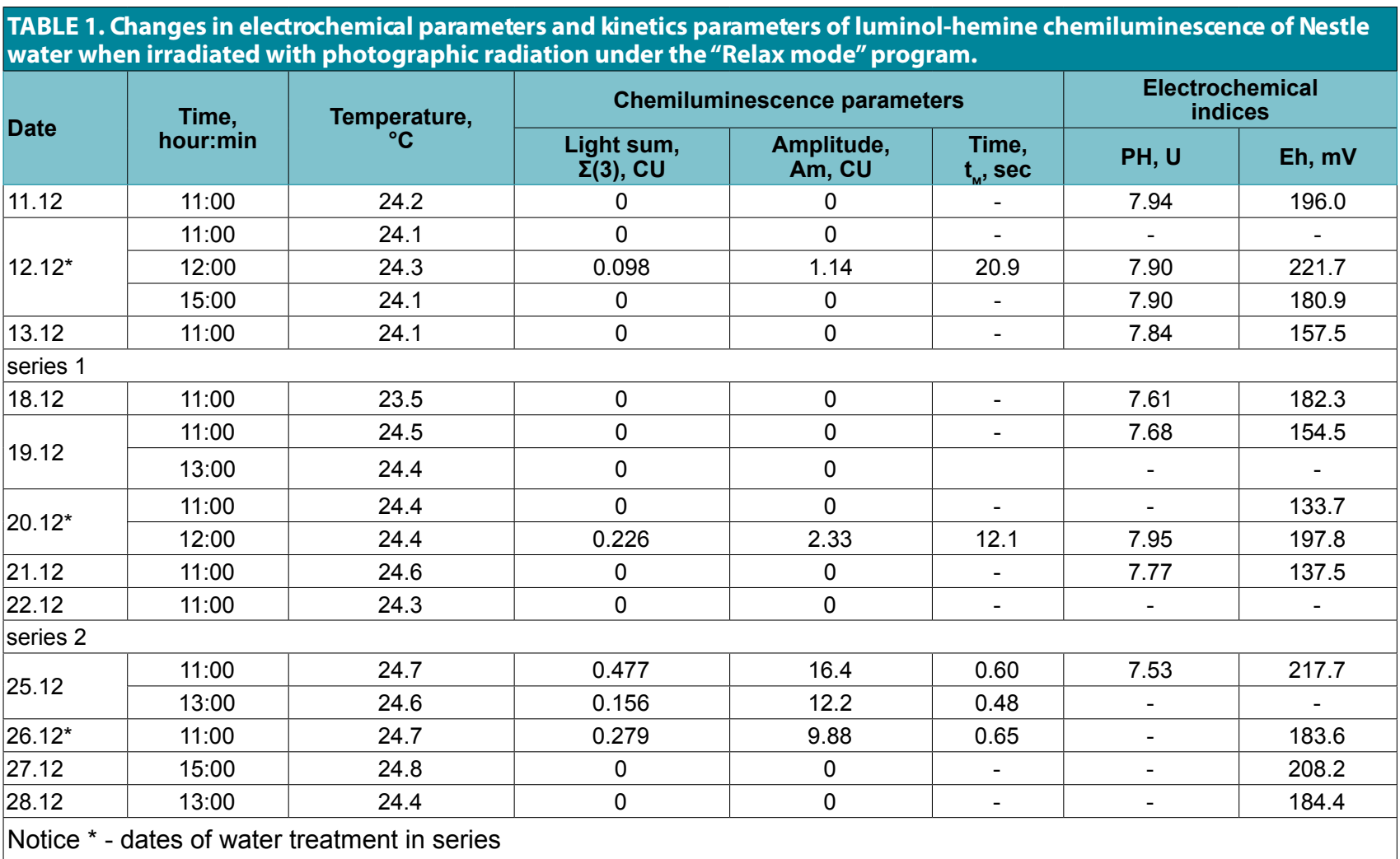


of water subjected to short-term irradiation with biologically active radiations of the visible wavelength spectrum allow us to assume both the presence of structural and physical changes in the associated water phase and the timeless interactions of radiation-excited associated water phase quantum states.

However, changes in the water electrochemical parameters are observed as early as 6 days before treatment. This suggests the occurrence of electrochemical processes in water, which are initiated by the forthcoming modulation (non-locality of the $2^{\text {nd }}$ class [18]).

The assessment of structural and energetic changes in water was carried out daily for 10 days with 2 series (TABLE 2).

Analysis of the data in TABLE 2 indicates the dynamic changes in the "Nestle" water associates structural organization, exposed to irradiation under the "Relax mode". Changes in the water associates structural organization can be observed by the decrease of phase fraction values in the 1-2 hours after the water treatment and by their energy levels redistribution. Changes in the water associates structural organization are accompanied by a phase fraction decrease in 1-2 hours after water treatment, and their redistribution by energy levels.

Thus, after the water irradiating, the energy level population $\mathrm{q} i=0.4 \ldots 0.6 \%$ compared to untreated water decreases in both experiments series. Upon that, the process of associated water phase structural organization transformation $(\mathrm{qi}=0.4 \ldots 0.6 \% \rightarrow \mathrm{qi}=0.6 \ldots 0.8 \%)$ proceeds at a time-increasing rate, and only after two days of the water holding in a stable state, the phase changes transformation towards the low-energy level population. This process proceeds through the stages of states population with a smaller and simultaneously higher energy $\left(\mathrm{q}_{\mathrm{i}}=0.6 \ldots 0.8 \%\right.$ $\rightarrow \mathrm{q}_{\mathrm{i}}=0.2 \ldots 0.4 \%$ and $\left.\mathrm{q}_{\mathrm{i}}=0.8 \ldots 1.0 \%\right)$.

However, it should be noted that the structural transformation of the phase takes place not only "in direct" time, but also reveals itself in advanced states 1 and 2 days before the water irradiation (TABLE 2 and FIGURES 1-3). The anomalous increase in the associated water phase fraction values of the 2 days before the irradiation $\left(\mathrm{q}_{\mathrm{av}}=0.494 \%\right.$ - the $1^{\text {st }}$ series and $\mathrm{q}_{\mathrm{av}}=0.551 \%$ - the $2^{\text {nd }}$ series) compared to the control $\left(\mathrm{q}_{\mathrm{av}}=0.3950 .442 \%\right)$ is a significant value that cannot be attributed to experimental error. Moreover, a stronger population of the energy level $\mathrm{q}_{\mathrm{i}}=0.4 \ldots 0.6 \%$ is observed $\left(\Delta \mathrm{q}_{\mathrm{i}}=0.4 \ldots\right.$ $0.6 \%=0.55 \ldots 0.6$ rel.-in series 1 and 2 against $\Delta q_{i}=0.4 \ldots 0.6 \%=0.250 .40$ rel. - the control).

The dynamics of water structural organization exposed to radiation is clearly revealed in the temporal trend of the change in the associated water phase fraction (FIGURE 1). These changes reach extreme values on the day after the irradiation (minimum) with the appearance of two maxima ( 1 day before irradiation and 2 days after water irradiation). The trend is almost identical in both measurements series.

In graphical form, the transformation of water associates energetic distribution (FIGURES 2 and 3) has an unfinished wavelike character, which suggests a further evolution of the distribution that extends beyond the time limits of two daily experiments intervals.

\begin{tabular}{|c|c|c|c|c|c|c|c|c|c|}
\hline \multirow{2}{*}{ Date* $^{*}$} & \multirow{2}{*}{ Temperature, $\mathrm{T},{ }^{\circ} \mathrm{C}$} & \multicolumn{2}{|c|}{ Phase fraction } & \multicolumn{6}{|c|}{ Energy level, $q_{i} \%$} \\
\hline & & $\mathrm{q}_{\mathrm{av}}, \%$ & $\operatorname{var}(q)$ & $0-0.2$ & $0.2-0.4$ & $0.4-0.6$ & $0.6-0.8$ & $0.8-1.0$ & $1.0-1.2$ \\
\hline 17.10 & 23.1 & 0.395 & 0.041 & 0.05 & 0.45 & 0.40 & 0.10 & 0 & 0 \\
\hline 14.11 & 23.5 & 0.442 & 0.038 & 0.05 & 0.35 & 0.25 & 0.25 & 0.1 & 0 \\
\hline \multicolumn{10}{|c|}{ series 1} \\
\hline 19.12 & 23.7 & 0.494 & 0.025 & 0.05 & 0.1 & 0.6 & 0.2 & 0.05 & 0 \\
\hline $20.12^{*}$ & 23.8 & 0.48 & 0.021 & 0 & 0.3 & 0.55 & 0.1 & 0.05 & 0 \\
\hline 21.12 & 23.9 & 0.382 & 0.047 & 0.15 & 0.35 & 0.4 & 0.05 & 0.05 & 0 \\
\hline 22.12 & 24.4 & 0.526 & 0.020 & 0 & 0.2 & 0.35 & 0.45 & 0 & 0 \\
\hline \multicolumn{10}{|c|}{ series 2} \\
\hline 25.12 & 23.9 & 0.551 & 0.027 & 0.05 & 0.1 & 0.55 & 0.2 & 0.05 & 0.05 \\
\hline $26.12^{*}$ & 22.6 & 0.483 & 0.023 & 0 & 0.2 & 0.65 & 0.1 & 0.1 & 0 \\
\hline 27.12 & 23.5 & 0.413 & 0.028 & 0.1 & 0.35 & 0.4 & 0.2 & 0 & 0 \\
\hline 28.12 & 22.9 & 0.576 & 0.017 & 0.05 & 0.1 & 0.35 & 0.5 & 0.05 & 0 \\
\hline 29.12 & 23.4 & 0.585 & 0.027 & 0 & 0.15 & 0.35 & 0.4 & 0.15 & 0 \\
\hline
\end{tabular}




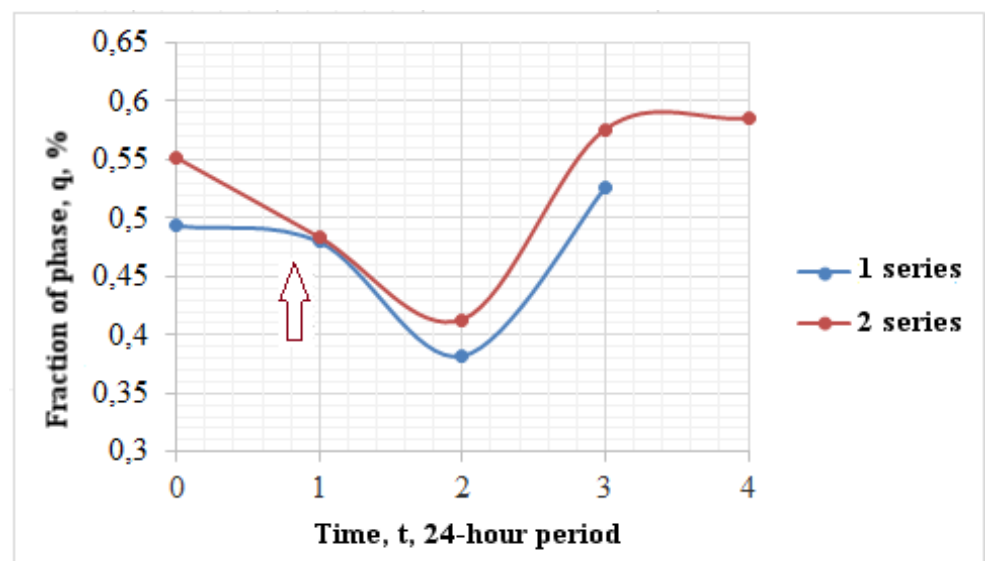

FIGURE 1. Changes in the associated water phase fraction $\left(q_{\mathrm{av}}, \%\right)$ in Nestle water in weekly periods of exposure (before and after 6 minutes of treatment with radiation under "Relax mode" in factory container).

FIGURE 2. Changes in the associated water phase population difference $\left(\Delta q_{i}=0.2-0.6 \%\right.$ $\left.\Delta q_{i}=0.6-1.0 \%\right)$ in Nestle water during weekly exposure time periods (before and after 6 minutes of treatment with radiation under the "Relax mode" in factory containerindicated by the arrow).
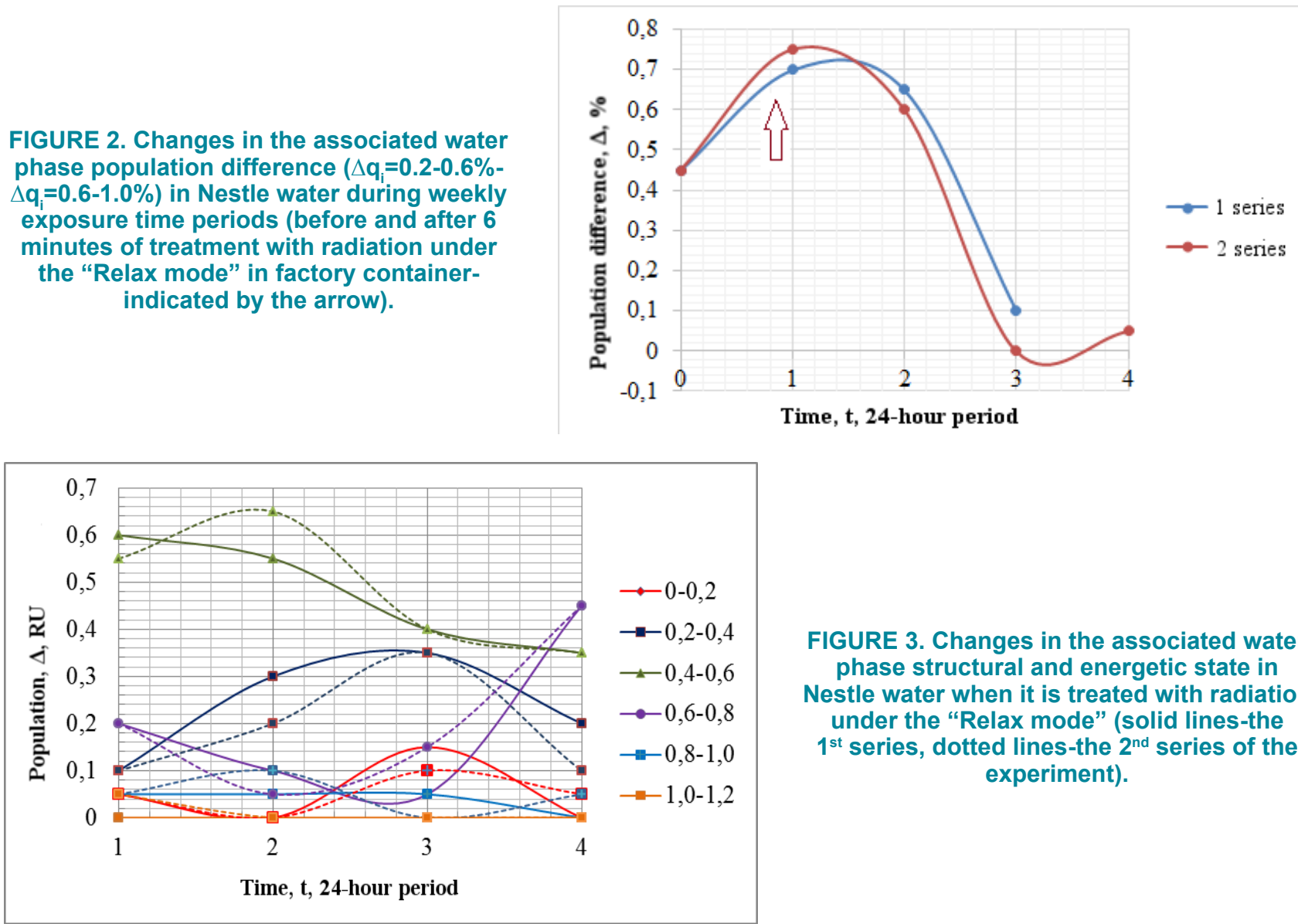

FIGURE 3. Changes in the associated water phase structural and energetic state in Nestle water when it is treated with radiation under the "Relax mode" (solid lines-the $1^{\text {st }}$ series, dotted lines-the $2^{\text {nd }}$ series of the experiment).

It is likely that the driving force of nontemporal water associates energetic distribution transformation is perturbations of the electrons Bose condensate, which have nonlocal responses not only in space but also in time.

Generalizing the trends in the associated water phase structural organization during its photomodulation using the Relax mode, one can note the same tendency for the associated water phase population levels to shift to low values $\left(\mathrm{q}_{\mathrm{i}}=0.2-0.6 \%\right)$ when the water is treated, which in the following days is transformed in the area of higher values of $\mathrm{q}_{\mathrm{i}}=0.6-1.0 \%$.

The change in the water associates energetic distribution under the influence of modulated light radiation (Relax mode) affects the biological activity of test organisms (FIGURE 4) [16]. Thus, for the ("Ecolum" test culture, the stimulating effect of the water associates is mainly revealed at an energy level of $\mathrm{q}_{\mathrm{i}}=0.4$ ... $0.6 \%$; whereas, the water associates in the 


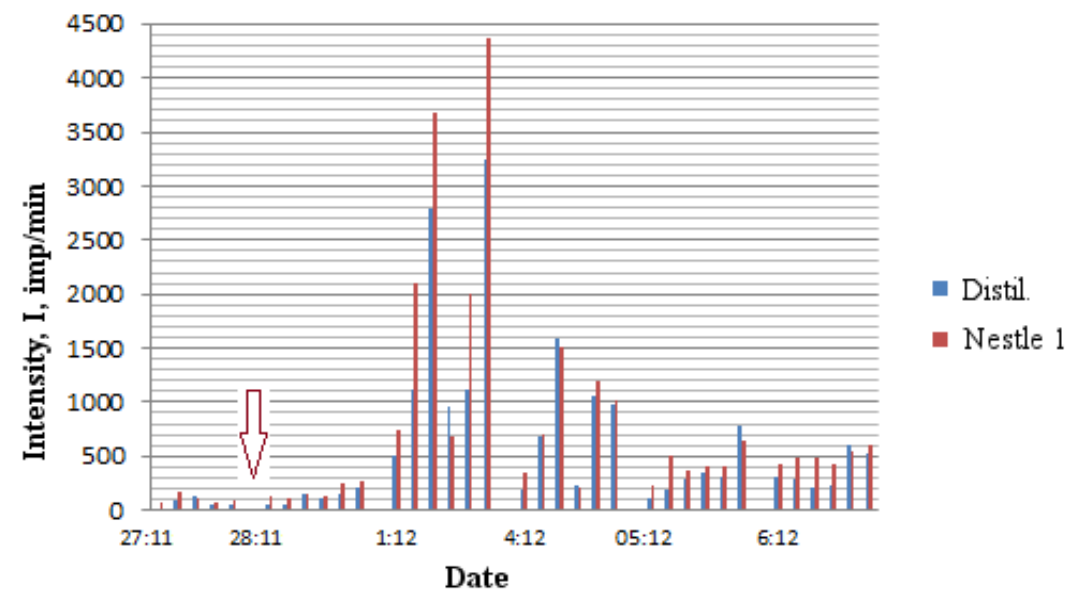

range of the structural organization $\mathrm{q}_{\mathrm{i}}=0.6$ ... $0.8 \%$ have a suppressive effect on the growth and development of E. coli ("Ecolum") microorganism.

The study of photomodulated water effect on the test culture biological activity has established that microorganisms react nonlinearly to changes in the structural and energetic state of the aquatic environment. This reaction of the test culture is manifested in delayed (for 2 or more days) or in advance (for two days) time intervals. What is observed is a slight decrease in the microorganisms activity on the day of exposure and on subsequent days (TABLE 3).

The correlation analysis between the activity of "Ecolum" microorganisms and the associated water phase energetic distribution (TABLE 4) indicates the transformation of its resonant states, which determine the microorganisms culture development [12]. The resonance level of life activity stimulation is represented by hydroxyl associates in the range of states $\mathrm{q}_{\mathrm{i}}=0.4$ ... $0.6 \%$, with moves to the range of $\mathrm{q}_{\mathrm{i}}=0.6 \ldots$ $0.8 \%$. Therefore, the energy level of peroxide associates $\left(\mathrm{q}_{\mathrm{i}}=\begin{array}{lllllll}0.8 & \ldots & 1.0 \% & \mathrm{q}_{\mathrm{i}}=0.4 & \ldots & 0.6 \%\end{array}\right)$, stimulating the activity of microorganisms, is practically weakly affected, this reaction is obviously associated with their lesser resistance.

It is obvious that the water excitation associates by long exposure to radiation lead to a change in the binding energy of electrons in the amorphous ice matrix, which in turn changes the conditions of macroscopic quantum interaction with the organism's structures. This implies the need for further studies the water associates matrix resonance properties with human body systems.
Figure 4. The dynamics of "Ecolum" test culture activity when irradiating Nestle water with optical radiation under the "Relax mode".
In general, the conducted series of studies confirm the significant light modulated influence in the "Relax mode" on the water structural and energetic state and its biological activity. However, this effect is not limited to a short-term exposure to the radiation source but affects long-term time intervals in both the delayed and the current phase activation states. It is obvious that the fact of nontemporal effect appearance of optical radiation on the associated water phase structural organization is related to the system quantum nature determined by the superfluid state of its delocalized electrons [3].

The study of the relationship between the reaction of human body systems and changes in the structural and energetic state of water.

The color correction program (yellow, green, lilac), which does not have a passive effect on the melatonin synthesis in the pituitary gland also shows a sympathetic-tonic effect on the human body, is used in the photo correction program in the "Relax mode" is intended for the body physiological relaxation (removal of mental and muscular tension). In this program, the green light has a calming effect on the body [17], lilac reduces the central nervous system (CNS) activity, and yellow has a normalizing effect on the respiratory system and a stimulating effect on the gastrointestinal tract function and pancreas [13].

In the unperturbed phase state, there is an inverse relationship, characterized by a correlation coefficient close to -1.0 , between the associated water phase fraction and the stress index of the volunteer's body, which was determined by the contact (through the hand) reactions of the body to water. The short-term water photomodulation (under the "Relax 
mode" program) leads to the initiation of associated water phase structural organization transformation. Therefore, the phase passes to the state of accumulation and simultaneous binding of electrons, which is revealed in the increase the body's regulatory systems stress index (FIGURE 5).

Thus, the untreated water effect (1 week before the water irradiation) is characterized by a correlation coefficient between the associated water phase fraction and the reaction of the body, which has a value $\mathrm{C}_{\text {corr }}=-0.95$. One day before the treatment and on the treatment day, the correlation coefficient between these parameters acquires a positive value (Correction $=+1.0$ ). However, between 2 and 3 , the correlation coefficient returns to the correlation values that are characteristic the untreated water. Such a change in correlation characteristics corresponds to changes in biological activity.
During the study it has been established that the more time passes after the water treatment, the more significant changes are noted in the state of the water structural organization and in the values of the body's regulatory systems stress index.

When estimating the effect of water photomodulation on the body's regulatory systems, it should be noted that these changes in the water state lead to a decrease in the $\mathrm{Si}$ values to a level close to the optimal level ( $\triangle \mathrm{Si}=-190,000$ units). The phase fraction does not undergo major changes and remains at the level of the initial values equal to $\mathrm{q}_{\mathrm{av}}-0.480 \%$. The human- unfriendly deformation of the water structural and energetic state treated with radiation appears at certain time periods after 3 or more days after the water photomodulation $(\mathrm{Si}-650)$, this is associated with a decrease in the water electron-doping ability.

\begin{tabular}{|c|c|c|c|c|c|}
\hline \multicolumn{3}{|l|}{ Series 1} & \multicolumn{3}{|c|}{ Series 2} \\
\hline Date & lav.d. & $\Delta \mathbf{q}, \%^{*}$ & Date & lav.d. & $\Delta \mathbf{q}, \%^{*}$ \\
\hline 18.12 & 1918.6 & - & 25.12 & 576.3 & 0.45 \\
\hline 19.12 & 464.4 & 0.45 & $26.12^{\star *}$ & 530.8 & 0.75 \\
\hline $20.12^{\star *}$ & 461.8 & 0.7 & 27.12 & 279.5 & 0.6 \\
\hline 21.12 & 388.1 & 0.65 & 28.12 & 773.5 & 0 \\
\hline 22.12 & 255.4 & 0.1 & 29.12 & 660.4 & 0.05 \\
\hline
\end{tabular}

TABLE 4. Correlation coefficients of the "Ecolum" microorganisms activity $\left(\mathrm{I}_{\text {avd }}\right)$ with the energy levels population $\left(\Delta_{\mathrm{ai}}, \mathrm{RU}\right)$ in two consecutive series of "Nestle" water photomodulation (under the "Relax mode" program).

\begin{tabular}{|c|c|c|c|c|c|}
\hline \multirow{2}{*}{ Number of series } & \multicolumn{5}{|c|}{ Energy level $\mathbf{q}_{\mathbf{i}}, \boldsymbol{\%}$} \\
\cline { 2 - 6 } & $0-0.2$ & $0.2-0.4$ & $0.4-0.6$ & $0.6-0.8$ & $0.8-1.0$ \\
\hline $1(19-21.12)$ & 0.142 & -0.028 & 0.905 & -0.775 & 0.933 \\
\hline $2(25-28.12)$ & -0.505 & -0.917 & -0.102 & 0.702 & 0.505 \\
\hline
\end{tabular}

FIGURE 5. Long-term dynamic changes in the associated water phase fraction $\left(q_{a v}.\right)$ in Nestle water and Stress Index of volunteer's regulatory systems (Si) at contact testing. The positive values of the correlation coefficient $\mathrm{C}_{\text {corr }}$ (between Si and $q_{a v^{*}}$ ) correspond to the state of the associated water phase self-organization (set of electrons) and the negative values correspond to the phase of the electron emission.

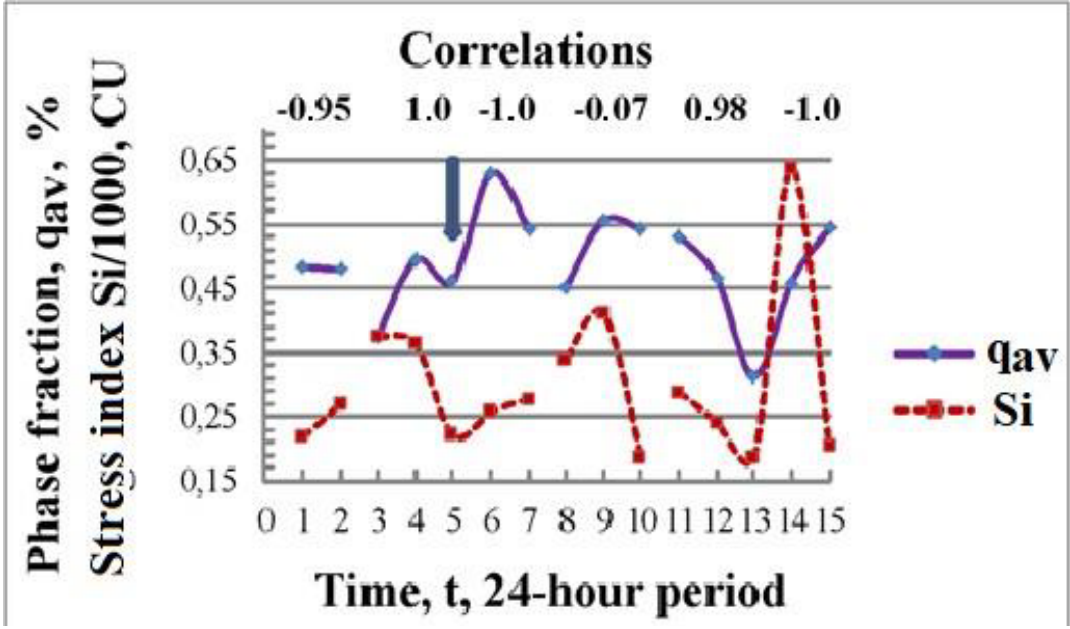


Thus, the phase of the associated water in the cellular structures serves as the primary link in the systemic body's homeostasis regulation determined primarily by its electrophysical state. The body's regulatory systems reaction to changes in the water structural and energetic state is nonlinear and generalized and encompasses its systems, which is determined by the quantum properties of the nonlocal spatial and temporal interaction of the associated water phase with the body cellular structures. Thus, the directivity of the electronic interaction is based on structural and electronic states selfsimilarity principles between the donor and the acceptor.

The associated water phase energy distribution (TABLE 5) is subject to strong changes after photomodulation.

The dynamics of the associated water phase structural and energetic organization is consistent with the changes noted above, as presented in FIGURES 1-3, indicating the redistribution of associates towards high-energy states.

Based on the dynamic changes results in the water structural and energetic state being studied under the photomodulation influence (TABLE 5) and the response of the volunteers' systems to the water (TABLE 6), a matrix of associated water phase resonant states the relative to organs and human body systems was developed. The matrix also illustrated the transformation of water under the photomodulation effect in the "Relax mode" (TABLE 7).

As the TABLE 6 data illustrates, the body systems reaction to the water photomodulation is characterized by a targeting change in its activity. Thus, the sympathetic-tonic effect of the "Relax mode" program emission on the human body is revealed in the decrease of the central nervous system activity on the day the water was treated. However, there is an opposite effect of the targeting of this influence, both 1 day before the water treatment and 1 day after the irradiation. During this timeframe, the central nervous system activity increases dramatically. Moreover, the central nervous system activity can detect the presence of a mirror the central nervous system's reaction symmetry during a 5-day measurements cycle (TABLE 6). This serves as an additional argument in favor of the water nontemporal reaction regularity to radiation (on the structural and energetic basis and electrochemical indicators).

The immune and venous systems, as well as the gall bladder, show similar changes in activity. Therefore, other systems that are opposite in sign, also symmetrized in time, exhibit dynamic changes (TABLE 6). The targeting of changes in the human body systems activity during water photomodulation under the "Relax mode" program fully corresponds to the previously established response of the body systems to direct

Table 5. Changes in the structural and energetic states (the fraction of the associated water phase $q_{a v}$ and the phase states population density $\Delta, \mathrm{RU}$ ) of Nestle water when it is activated by the light range of emissions under the "Relax mode".

\begin{tabular}{|c|c|c|c|c|c|c|c|c|}
\hline \multirow[t]{2}{*}{ Date } & \multicolumn{2}{|c|}{ Phase fraction qcp, \% } & \multicolumn{6}{|c|}{ Energetic levels $q_{i}, \%$} \\
\hline & average & variations & $0-0.2$ & $0.2-0.4$ & $0.4-0.6$ & $0.6-0.8$ & $0.8-1.0$ & $1.0-1.2$ \\
\hline 24:01 & 0.483 & 0.022 & 0 & 0.20 & 0.50 & 0.30 & 0 & 0 \\
\hline $25: 01$ & 0.479 & 0.019 & 0 & 0.30 & 0.60 & 0.10 & 0 & 0 \\
\hline 29:01 & 0.374 & 0.032 & 0.05 & 0.45 & 0.40 & 0.10 & 0 & 0 \\
\hline $30: 01$ & 0.494 & 0.027 & 0.05 & 0.15 & 0.5 & 0.25 & 0.05 & 0 \\
\hline $31: 01^{*}$ & 0.461 & 0.029 & 0.05 & 0.35 & 0.35 & 0.25 & 0 & 0 \\
\hline 1:02 & 0.63 & 0.019 & 0 & 0.15 & 0.25 & 0.45 & 0.10 & 0.05 \\
\hline $2: 02$ & 0.543 & 0.020 & 0 & 0.15 & 0.55 & 0.15 & 0.15 & 0 \\
\hline $7: 02$ & 0.556 & 0.017 & 0 & 0 & 0.70 & 0.25 & 0.05 & 0 \\
\hline 8:02 & 0.541 & 0.020 & 0 & 0.20 & 0.30 & 0.50 & 0 & 0 \\
\hline $12 \cdot 02$ & & & & & & & & \\
\hline 12.02 & 0.532 & 0.021 & 0.05 & 0.20 & 0.40 & 0.30 & 0.05 & 0 \\
\hline 13:02 & 0.467 & 0.022 & 0 & 0.30 & 0.55 & 0.01 & 0.05 & 0 \\
\hline 14:02 & 0.312 & 0.036 & 0.15 & 0.60 & 0.25 & 0 & 0 & 0 \\
\hline 15:02 & 0.459 & 0.037 & 0 & 0.40 & 0.35 & 0.15 & 0.05 & 0.05 \\
\hline 16:02 & 0.545 & 0.021 & 0 & 0.10 & 0.60 & 0.20 & 0.01 & 0 \\
\hline
\end{tabular}

Notice *- the date of water treatment 


\begin{tabular}{|c|c|c|c|c|c|c|c|c|c|c|c|c|}
\hline \multirow[t]{2}{*}{ Date } & CNS & \begin{tabular}{|c|} 
Endocrine \\
system
\end{tabular} & $\begin{array}{c}\text { Respiratory } \\
\text { system }\end{array}$ & Stomach & cVs & $\begin{array}{c}\text { Intestinal } \\
\text { tract }\end{array}$ & Kidneys & $\begin{array}{c}\text { Reproductive } \\
\text { system }\end{array}$ & Liver & Bladder & $\begin{array}{l}\text { Venous } \\
\text { system }\end{array}$ & $\begin{array}{l}\text { Immune } \\
\text { system }\end{array}$ \\
\hline & 1 & 2 & 3 & 4 & 5 & 6 & 7 & 8 & 9 & 10 & 11 & 12 \\
\hline 24.1 & 12.12 & -3.12 & -4.44 & -1.2 & 0.36 & 2.04 & -4.68 & -4.8 & -2.16 & 0 & -0.6 & 7.44 \\
\hline 25.1 & 5.88 & -1.08 & -2.28 & -11.4 & -3.6 & 1.56 & -5.64 & -6.6 & 5.28 & 13.44 & 4.8 & 5.16 \\
\hline 29.1 & -5.52 & 5.16 & 13.68 & 0.24 & -3.72 & -12 & -0.6 & -132 & 228 & -2.04 & -4.92 & 324 \\
\hline 30.1 & 23.76 & 4.2 & -24.24 & -6.6 & -4.2 & -9.72 & -8.88 & -5.04 & 0.24 & 0 & 10.56 & 13.8 \\
\hline 31.1 & 2.04 & 5.16 & 5.76 & 14.16 & 6.36 & -6.12 & -0.36 & 9.24 & -3.84 & -8.4 & -7.08 & -10.44 \\
\hline 1.2 & 20.76 & 3.48 & -11.28 & -2.4 & -11.52 & -10.32 & -0.12 & 6.24 & -0.96 & -0.72 & 2.28 & 3.12 \\
\hline 2.2 & -6.84 & 7.68 & 7.44 & 1.8 & 3.12 & 1.68 & 5.52 & 9 & 1.08 & -5.76 & -11.28 & -9.84 \\
\hline 62 & 1032 & 192 & 1236 & 828 & 18 & 110 & 1068 & 624 & 768 & 024 & 1632 & -2136 \\
\hline 7.2 & -16.92 & -2.04 & 25.44 & 15.36 & -8.88 & -10.8 & 20.64 & 19.56 & -13.08 & -9.12 & -12.72 & -19.68 \\
\hline 8.2 & 12.12 & 2.52 & -7.08 & -6.36 & -1.56 & $\begin{array}{l}-1.8 \\
\end{array}$ & 2.28 & 7.8 & 3.12 & -7.32 & -2.4 & 4.68 \\
\hline & & & & & & & & & & & & \\
\hline 12.2 & -3.24 & 0.72 & -6.84 & -4.92 & 6 & -0.96 & 2.76 & 8.52 & 13.56 & 4.56 & -11.04 & -12.36 \\
\hline 13.2 & 17.4 & 0.84 & 10.92 & 8.52 & -6.24 & -17.4 & -6.72 & -0.96 & -3 & 1.8 & -4.2 & 8.76 \\
\hline 14.2 & -3.48 & 10.2 & -4.2 & -14.88 & -3.48 & 11.16 & -0.36 & -10.92 & 0.12 & 1.2 & 7.32 & 5.76 \\
\hline 15.2 & -5.4 & -6.36 & -6.6 & -9.24 & -1.8 & -1.92 & 1.08 & -1.56 & -0.96 & -0.84 & 15.6 & 16.68 \\
\hline 16.2 & -2.4 & 5.76 & -11.16 & -9.96 & 3.96 & -0.72 & -6.48 & -5.28 & 6.36 & 2.28 & 10.56 & 10.68 \\
\hline
\end{tabular}

Notice * $^{*}$-difference between the background value and water, A, \%; water treatment-31.1; significant (being higher than the value of dispersion of signals) values of human body reaction (on average volunteer data) are shown in bold.

Table 7. Resonance states of the associated water phase energy levels relative to the organs and systems of the human body and their transformation upon water irradiation under the "Relax mode"

\begin{tabular}{|c|c|c|c|c|c|c|}
\hline \multirow{2}{*}{ Human body systems } & \multicolumn{6}{|c|}{ Energy levels, $q_{i}$} \\
\hline & $0-0,2$ & $0,2-0,4$ & $0,4-0,6$ & $0,6-0,8$ & $0,8-1,0$ & $1,0-1,2$ \\
\hline Central nervous system, & & & & & & \\
\hline Endocrine system, & & & & & & \\
\hline $\begin{array}{l}\text { Respiratory system, autonomic } \\
\text { nervous system, }\end{array}$ & & & & & & \\
\hline Digestive system, stomach, & & & & & & \\
\hline Cardiovascular system, & & & & & & \\
\hline Digestive system, intestinal tract, & & & & & $>$ & \\
\hline Excretory system, kidneys, & & & & $>$ & & \\
\hline Reproductive system, & & & & & & \\
\hline Arterial system, liver, & & & $<$ & & & \\
\hline $\begin{array}{l}\text { Musculoskeletal system, gall } \\
\text { bladder, }\end{array}$ & & & & & & \\
\hline Venous system, & & & & & $\Rightarrow$ & \\
\hline Immune system, lymphatic system, & & & & & & \\
\hline
\end{tabular}

radiation. Thus, the central nervous system reduces its activity against the background of the normalization of the respiratory organs and stimulation of the gastrointestinal tract with simultaneous cardiovascular system improvement. This indicates that the primary link of the radiation regulatory action on the human body is the associated water phase both inside and outside the body.

Changes in the human body systems resonance states (TABLE 7), the energy of the associated water phase distribution (TABLE 5) allows one to determine the targeting transformation of human body system activity 
upon the water treatment by radiation under the "Relax mode" program. Thus, the systems presented in TABLE 7, experiencing the inhibitory effect of radiation-treated water, changes their resonant (stimulating) state, the number $(\Delta, \mathrm{R})$ of which is less than on previous day:

- immune system, gall bladder $\mathrm{q}_{\text {stim }}=0.4-0.6 \%-\Delta_{\text {before }}=0.25, \Delta_{\text {after }}=0.175$;

- venous system - $\mathrm{q}_{\text {stim }}=0.8-1.0 \%$ $\Delta_{\text {before }}=0.025, \Delta_{\text {after }}=0$;

- central nervous system - $\mathrm{q}_{\text {stim }}=0.6-0.8 \%$ $\Delta_{\text {before }}=0.125, \Delta_{\text {after }}=0.125$;

The increasing systems activity (the respiratory, stomach, reproductive one) as a result of water irradiation is mainly characterized by the phase energy level $(\mathrm{q}=0,2-0,4 \%)$, which increases $\left(\Delta_{\text {before }}=0.075, \Delta_{\text {after }}=0.16\right)$ after the light correction, except for the cardiovascular system $\left(q_{\text {stim }}=0.4-0.6 \%\right)$, the activity of which is likely to increase due to intersystem interactions.

The relative contribution of inter-system interactions regulated by the central nervous system activity to the primary adaptation reaction and a specific response to water (targeting), histograms of changes in the body systems activity the and the water resonant energy levels population exposed to light irradiation (under the "Relax mode" mode) were developed. The comparison of these parameters in the eustress response (from 30.01 to 31.01-FIGURE 6) allows for the establishment of systems that react to the associated water phase under the balanced mode as well as the systems that change their state on the basis of direct intersystem connections.

The body's reaction to eustress (decrease in the values of the stress index of regulatory systems - see FIGURE 5) is characterized by specific responses (targeting) of water in respect to the digestive, respiratory, immune, reproductive and venous systems, which is determined by the high value of the cross-correlation coefficient between the change in the response of systems and the change in the associated water phase energy levels population $\left(\mathrm{C}_{\text {corr }}=0.967\right)$.

Upon the body stress reaction, the body
FIGURE 6. Changes in the response of the regulatory activity of volunteers' body systems to the gradient of the resonant energy level population (according to the table) of "Nestle" water in 30.01-31.01 period, subjected to radiation treatment under the "Relax mode" program (eustress response to water activation).

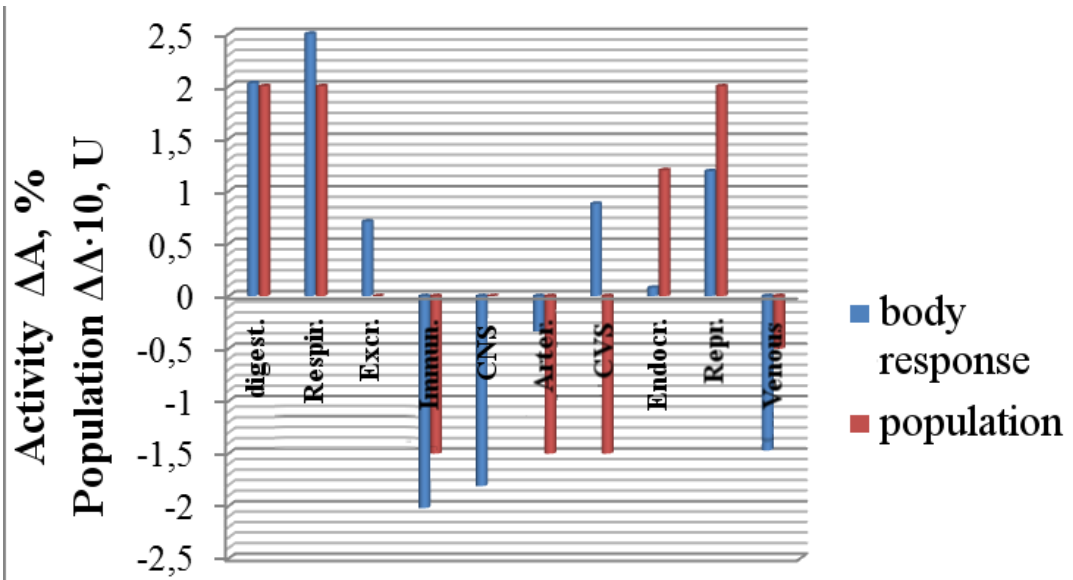

FIGURE 7. Changes in the response of volunteers' body systems regulatory activity to the gradient of the resonant energy level population (according to the table) of "Nestle" water in 31.01-01.02 period, subjected to radiation treatment under the "Relax mode" program (stress response to water activation). 
systems targeting is transferred to the digestive, respiratory, venous, cardiovascular and central nervous systems $\left(\mathrm{C}_{\text {corr }}=0.968\right)$ (FIGURE 7). The reaction of other body systems (both under eustress and under stress) is characterized by low values of the correlation relationship, which indicates its dependence on intersystem interactions.

The structural scheme (FIGURE 8) reflects the interaction between individual systems and organs of the body.

The targeting of body systems specific indications involves other systems in the adaptation process by the principle of interaction with them, toning or inhibiting activity. Under stress, the system with the highest reaction rate contributes to excessive inhibition of the other

body systems functioning by its high sedative activity. Thus, the systems experiencing a tonic effect show excessive activity, which determines their reaction under Le ChatelierBraun principle.

The energy of the human body systems interconnection shown in FIGURE $\mathbf{8}$ is determined by two circuits: the left part is the doping energy circuit, functioning from the digestive system, the right part is the electronic circuit determined by the electrons influx to the central nervous system and the arterial system. Solid arrows denote the energy tonic relationship, and the dotted arrows denote the inhibitory energy relationship between the systems (circuits). The electronic circuit of the digestive system, adapted to the low-energy states of the associated water phase $\left(\mathrm{q}_{\mathrm{i}}=0.0\right.$ ... $0.4 \%$ ), is provided by the activity of the gastrointestinal microbiota, which is activated on the same resonant states of the phase [12].

The adaptation of CNS and arterial system to environmental conditions is due to bioenergetic phenomena that occur on the erythrocytes and leukocytes membranes [19] in the energy levels range of the associated water phase $\mathrm{q}_{\mathrm{i}}=0.6 \ldots$ $1.2 \%$ (TABLE 7), as shown by the presence of hydroxyl and peroxide associates. As the result of nonlocal quantum conjugation with electronic wavepackets of the phase (in external medium) enzymatic complexes of erythrocyte membranes provide a reversible binding of oxygen and carbon dioxide on the one hand, while on the other hand the condensation of electrons on paramagnetic oxygen centers, ensuring the electrons influx into the body and their further translation on conjugated systems occurs.

Water treated with light radiation under the

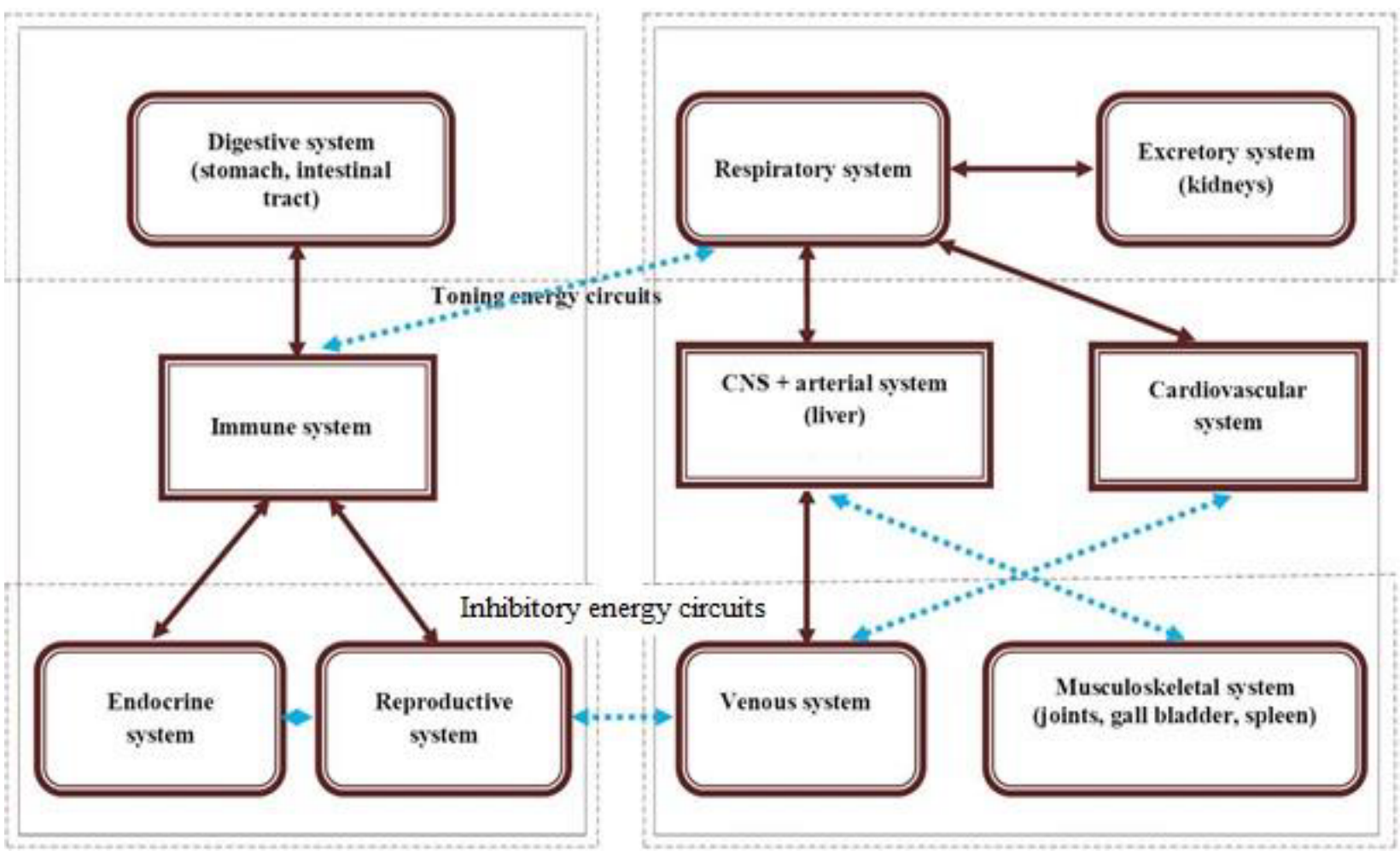

FIGURE 8. The interconnection of human body systems in a regulatory response to stress. 
"Relax mode program" (TABLE 6, data as of 31.01) has an inhibitory effect on CNS, leading to the decrease of its energy activity (before the treatment-23.76\%, after the treatment- +2.04 $\%)$. At the same time, the respiratory system toning (before the treatment-24.24, after the treatment-+5.76\%) and the venous system inhibition (before the treatment- +10.56 , after the treatment-7.08\%) takes place. Such energy redistribution in the body is caused by the change of electronic saturation in the associated water phase.

The cardiovascular system activation is carried out under the influence of three main systems: the respiratory and excretory ones (toning), and the venous one (sedation). These systems are energetically connected with CNS, the activity of which depends on the energy state of the external environment (water). The reduction of the water-energy state (at the resonant level) under the influence of treatment leads to the CNS activity decrease.
This reduces the inhibitory effect of the venous system with a slight decrease in the tonic effect on the respiratory system, which contributes to a greater energy intake by the cardiovascular system from the respiratory and excretory systems. This energy redistribution contributes to the increase of cardiovascular system energy activity (before the water treatment $-4.2 \%$, after treatment-6.36\%).

Thus, due to the energy redistribution through the body systems, there is an energy deficit compensation in body systems and organs, which demonstrates the inhibited activity state before the exposure to radiation.

To determine the difference in the effect of another water activation program (Energy), an additional series of water structural organization measurements were performed. The water treatment by various radiation programs (Relax, Energy) leads to changes in the associated water phase energy distribution, accompanied mainly by the redistribution of associates in the Energy
Figure 9. The dynamic dependence in the energy distribution (population of levels $\Delta$, RU) in the associated water phase under the photomodulation effect (program "Relax mode").

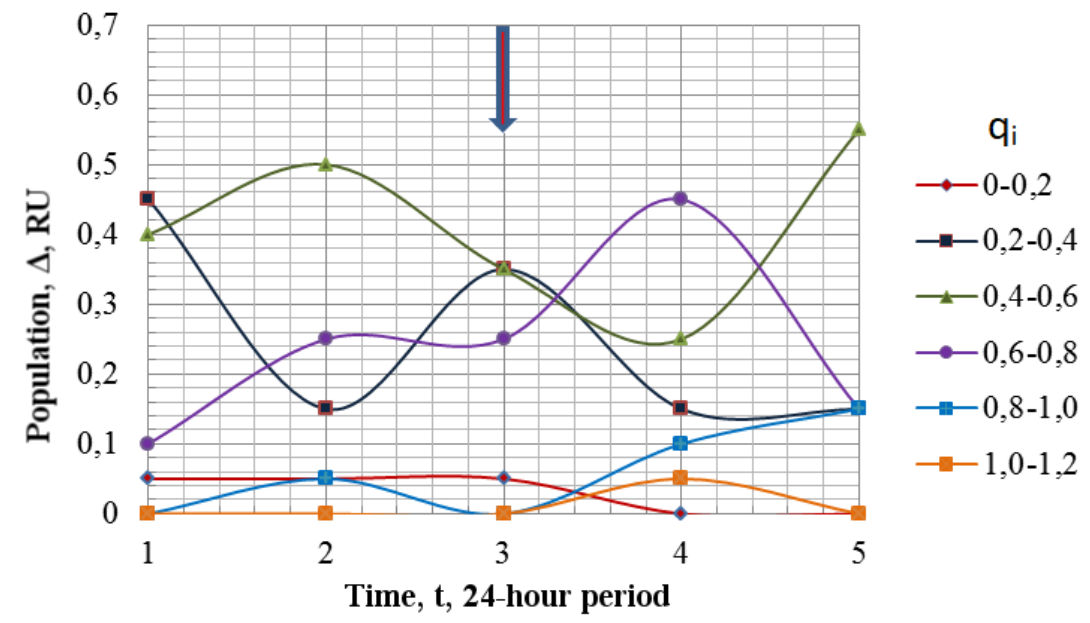

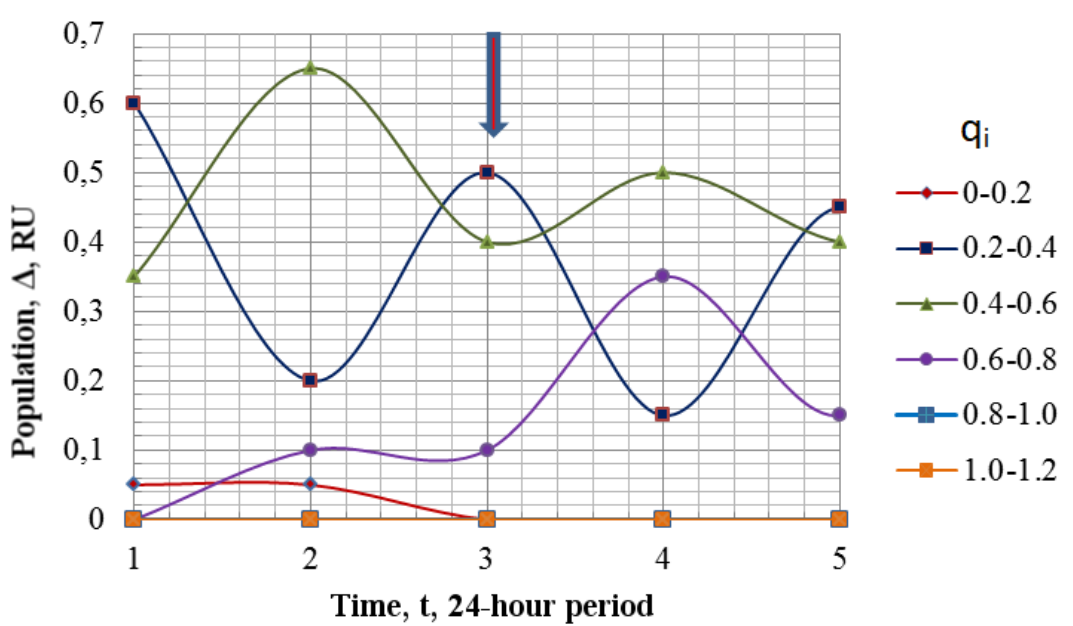

Figure 10. The dynamic dependence in the associated water phase energy distribution under the photomodulation effect (program the "Energy mode") 


\begin{tabular}{|c|c|c|c|c|c|}
\hline $\begin{array}{l}\text { TABLE 8. The di } \\
\text { phase energy le } \\
\text { the treatment of } \\
\text { mode" and the }\end{array}$ & $\begin{array}{l}\text { eren } \\
\text { els } \\
\text { Nes } \\
\text { Ener }\end{array}$ & $\mathrm{me}$ & $\begin{array}{l}\text { soc } \\
\text { on ( } \\
\text { er uI } \\
\text { e" p }\end{array}$ & $\begin{array}{l}\text { ed we } \\
\text { RU) b } \\
\text { er the } \\
\text { gram }\end{array}$ & $\begin{array}{l}\text { er } \\
\text { tween } \\
\text { Relax }\end{array}$ \\
\hline & Time & 24-ho & perio & & \\
\hline Energy level $\mathrm{q}_{\mathrm{i}}, \%$ & 1 & 2 & $3^{*}$ & 4 & 5 \\
\hline $0.0-0.6$ & -0.1 & -0.2 & -0.20 & -0.25 & -0.15 \\
\hline $0.6-1.2$ & 0.1 & 0.2 & 0.15 & 0.25 & 0.15 \\
\hline
\end{tabular}

program from level $\mathrm{q}_{\mathrm{i}}=0.6-0.8 \%$ to levels in the range of $\mathrm{q}_{\mathrm{i}}=0.2-0.6 \%$ (FIGURES 9 and 10 ).

Therefore, such changes in the phase structural organization not only influence the water on the day of the water treatment with radiation, but it also is redistributed for at least 5 days-symmetrically before the water treatment, on the day of exposure, and on subsequent days after the water treatment (TABLE 8). Similar aspects of change in the structural organization of the phase repeat the nonlocal dynamics of its structural state; this was noted during previous experiments. Some differences in the phase energy distribution dynamics related, however, not so much to the general picture of the phase behavior non-local processes, but as to the effects of the "compression" or the "stretching" in time, which is dependent on the water volume being treated [12].

The nature of changes in the phase energy distribution indicates the transformation of water targeting on the human body, which was acquired as a result of the water photomodulation under the "Energy mode" program. The reduction of high-energy phase states activity should lead to the activation of the endocrine, respiratory and digestive systems with a simultaneous decrease in the activity of the cardiovascular, venous and immune systems. Experimentally, based on previously established photomodulation patterns under the "Energy mode" program, the central nervous system is activated. However, its activation under stressresponse is regulated on the basis of nonspecific adaptation patterns.

\section{Conclusion and Future Studies}

Clinical and preventive use of photomodulation is becoming increasingly popular due to the availability of technology for widespread use and its safety and effectiveness. The present research allows for the formation of a scientific base for photobiomodulation. This is based on the established regularities governing the structural and energetic state of the associated water phase under the influence of certain light range emissions. The obtained regularities are based on quantum concepts of the associated water phase. This is based on the proof of the superconducting state of the phase electronic subsystem the and its ability to the nonlocal translation of its quantum state (de Broglie wavepacket of electrons) to the conjugate structures of the body systems, where they perform both energy [16] and regulatory functions [4]. These biological functions mediated by the effects of photostimulation [20] are associated with the charge activation of the bodywater [21-23] and subsequent incorporation of ATP F0F1 synthesis mechanisms by ATP synthase of cells mitochondria due to nonlocal electron intake from an external source $[3,16]$. Photobiomodulation of water excludes the use of high-intensity electromagnetic influences and is informational in nature, which is determined by the hypersensitivity of phase electrons superconducting state to the electromagnetic and acoustic radiation.

Changes in the state of the phase and, consequently, it's biological responses are strongly dependent on the duration and periodicity of irradiation, which is determined by the interference of nonlocal nontemporal excitations of the electronic phase subsystem under the unmodulated and modulated radiations action. In this regard, simultaneous irradiation of the body with many emission frequencies (antagonists) or the use of modulated EMW can cause opposite effects to the effects of unmodulated EMWs [24-26]. In this case, the multifrequency modulated photo irradiation over long periods has an after effect (with single photo irradiation), which can exacerbate the regulatory response of the body systems. This must be taken into account when prescribing phototherapy procedures [20,24].

Based on the results of the study, the clinical and prophylactic use of photobiomodulation programs should be based on the low-intensity multispectral regimes being strictly regulated in time and on the water exposure (human body) and adapted to the transformation of structural organization of the associated water phase, aimed at the sedation and stimulation of certain body systems, taking into account their intersystem interaction. 


\section{References}

Pollack GH, Figueroa X, Zhao Q. Molecules, water, and radiant energy: new clues for the origin of life. Int. J. Mol. Sci. 10(4), 1419-1429 (2009).

Paunesku T, Woloschak GE. Effects of radiofrequency and extremely lowfrequency electromagnetic field radiation on cells of the immune system in Biological and medical aspects of electromagnetic fields. ed. by F. Barus, B. Greenbaum. (2007).

Stekhin A, Yakovleva G, Pronko K, Zemskov V. Water is the main regulator of intracellular processes. Clin. Pract. 15(5), 841-855 (2018)

Stekhin A, Yakovleva G, Pronko K, Zemskov V. Regulatory function of macroscopic quantum states of electrons in cell metabolism. Clin. Pract. 15(3), 707-715 (2018)

Rakhmanin YA, Stekhin AA, Yakovleva GV. Biophysics of water: Quantum nonlocality in water treatment technologies; the regulatory role of associated water in cellular metabolism; rationing of bioenergetic activity of drinking water. Moscow, Publishing house Lenand. 353 (2016).

Rakhmanin $\mathrm{Yu}$ A, Kondratov VK, Mikhaylova RI, Stekhin AA, Yakovleva GV. Water is a cosmic phenomenon: cooperative properties and biological activity (the second edition, revised and supplemented). Karlovy Vary, CZ, Publishing house Tinowa. 457 (2014).

Zecha JA, Raber DJE, Nair RG. Lowlevel laser therapy/photobiomodulation in the management of side effects of chemoradiation therapy in head and neck cancer: Part 1: Mechanisms of action, dosimetric, and safety considerations. Support. Care Cancer. 24(6), 2781-2792
(2016).

Pavlov SE. Adaptation. M. Parusa, 282 (2000).

Marasanov AV, Valtseva EA. Phenomics. Etiology of functional states of the human body under the influence of environmental factors. Hygiene and sanitation. 96(10), 1004-1009 (2017).

Sudakov KV. General regularities of the dynamic organization of functional systems. K: Scientific and practical journal of the Kursk State Medical University, Central Black Earth Scientific Center of RAMS, Kursk regional branch of the Russian Academy of Natural Sciences "Man and his health". 2, 4-10 (2005).

Software "Rhythm-Express." User guide. LLC "Medical computer systems". Document number 4462.57 (2010).

Stekhin AA, Yakovleva GV. Structured Water: Nonlinear Effects. M.: LKI. 31 (2008).

Gotovsky SE, Vysheslavtsev AP, Kosareva LB. Color Phototherapy. M. Imedis. Russia. (2001).

Bansal N, Prakash NR, Randhawa JS, Kalra P. Effects of blue light on cognitive performance. Int. Res. J. Eng. Tech. 4(6), 434-2442 (2017).

Zhu L, Zee P. Circadian rhythm sleep disorders. Neurol. Clin. 30(4), 1167-91 (2012).

Stekhin AA, Yakovleva GV, Iksanova TI, Pronko KN, Zemskov VM. Evolution of the effect of structural-physical changes in water on biological activity. Clin. Pract. 15(5), 861-871 (2018).

Hirohiko F, Taku O, Yukiko I, Takuji $\mathrm{H}$, Takahisa S. Correction for 'Highefficiency ultrapure green organic lightemitting diodes. Mater. Chem.(2018).

Cramer JG. Transactional
Interpretation of Quantum Mechanics. Rev. Mod. Phys. 58, 647-688 (1986).

Borodyuk HP. Blood is a living being. Bioenergetic mechanisms of adaptive reactions. M. Globus. 214 (1999).

Tsai SRu, Hamblin MR. Biological effects and medical applications of infrared radiation. Journal of Photochemistry and Photobiology, B: Biology. 170, 197-207 (2017).

Chai B, Yoo H, Pollack GH. Effect of radiant energy on near-surface water. $J$ Phys Chem B. 113, 13953-13958 (2009).

Sommer AP, Haddad M, Fecht HJ. Light effect on water viscosity: implication for ATP biosynthesis. Sci. Rep. 5, 12029 (2015).

Passarella S, Karu T. Absorption of monochromatic and narrow band radiation in the visible and near IR by both mitochondrial and nonmitochondrial photoacceptors results in photobiomodulation. J. Photochem. Photobiol. B. Biol. 140, 344-358 (2014).

Tikhonov MN, Dovgusha VV, Dovgusha LV. The mechanism of the influence of natural and technogenic electromagnetic fields on the safety of vital activity. The Bulletin of Russian Academy of Natural Sciences. 18(4), 11-21 (2014).

Basar E, Guntekin B. Review of delta, theta, alpha, beta and gamma response oscillations in neuropsychiatric disordes. Application of brain oscillations in neuropsychiatric diseases. Suppl. Clin. Neurophysiol. 62, 303-341 (2013).

Monich VA, Malinovskaya SL, Mahrova TV, Malinovsky DS. Features of low-intensity electromagnetic radiation of various ranges on microorganisms. The Bulletin of Nizhny Novgorod University named after N.I. Lobachevsky. 2(2), 435438 (2010) . 